\title{
Pathological Renal Findings of Chronic Renal Failure in a Patient with the E66Q Mutation in the $\alpha$-galactosidase $A$ Gene
}

\author{
Atsushi Satomura ${ }^{1}$, Takayuki Fujita ${ }^{2}$, Tomohiro Nakayama ${ }^{1}$, Hiroyuki Kusano ${ }^{3}$, \\ Eiichi Takayama ${ }^{3}$, Hiroaki Hamada ${ }^{3}$ and Toshiharu Maruyama ${ }^{3}$
}

\begin{abstract}
A 66-year-old Japanese man was diagnosed with interstitial nephritis on a renal biopsy at 45 years of age and began to receive hemodialysis at 65 years of age. He was suspected of having Fabry disease as a result of a screening study for Fabry disease performed in hemodialysis patients. He had an E66Q mutation in the $\alpha$-galactosidase A gene. We conducted an electron microscopic examination of a renal biopsy specimen obtained when the patient was diagnosed with chronic renal failure at 45 years of age in order to elucidate the pathogenicity of the E66Q mutation. Interestingly, an electron microscopic examination of the renal biopsy specimen indicated no characteristic findings of Fabry disease.
\end{abstract}

Key words: Fabry disease, E66Q mutation, hemodialysis, renal biopsy, enzyme replacement therapy

(Intern Med 54: 1819-1824, 2015)

(DOI: 10.2169/internalmedicine.54.3279)

\section{Introduction}

Fabry disease (MIM 301500), also called Anderson-Fabry disease, is the second most prevalent lysosomal storage disorder after Gaucher's disease. Fabry disease is an X-linked hereditary disorder resulting from a deficiency of the $\alpha$ galactosidase A (GLA, EC 3.2.1.22) activity, which subsequently results in the accumulation of globotriaosylceramide (Gb3) and globotriaosylsphingosine within lysosomes in a wide variety of cells, thereby resulting in the protean clinical manifestations of the disease (1).

A deficiency of the GLA activity induces the progressive accumulation of glycosphingolipids, including $\mathrm{Gb} 3$, in various tissues, including the vascular endothelium, renal glomeruli and tubules, dorsal root ganglia, cardiac myocytes and heart valves, cornea and skin. Affected men with little or no detectable GLA activity exhibit a classic phenotype, with the onset of angiokeratoma, acroparesthesia and hypohidrosis in childhood. With advancing age, the development of renal failure, cardiac disease and stroke leads to a decline in activities of daily living and premature death. In contrast, patients with a substantial residual GLA activity demonstrate a milder late-onset phenotype, including renal (2) and cardiac (3) variants.

Generally, renal failure is a frequent cause of death in men with Fabry disease, whereas cardiac and cerebrovascular disease are the most common causes of death in affected women (4). According to the natural history of renal disease in patients with Fabry disease described by Branton et al. (5), the mean rate at which the glomerular filtration rate (GFR) decreases is $12.2 \pm 8.1 \mathrm{~mL} / \mathrm{min} / 1.73 \mathrm{~m}^{2} /$ year. A study by Feriozzi et al. (6) demonstrated that, although the mean yearly change in eGFR is $-2.2 \mathrm{~mL} / \mathrm{min} / 1.73 \mathrm{~m}^{2} /$ year in men and $-0.7 \mathrm{~mL} / \mathrm{min} / 1.73 \mathrm{~m}^{2} /$ year in women treated with enzyme replacement therapy (95\% confidence interval: -2.8 and $-1.7 ;-1.4$ and 0.0 , respectively), patients with a 24 -hour protein excretion of $>1 \mathrm{~g} / 24$ hours have a poorer renal function, as the mean eGFR decreases by $28.2 \pm 24.8 \mathrm{~mL} / \mathrm{min} /$ $1.73 \mathrm{~m}^{2} /$ year.

\footnotetext{
${ }^{1}$ Division of Laboratory Medicine, Department of Pathology and Microbiology, Nihon University School of Medicine, Japan, ${ }^{2}$ Division of Nephrology, Hypertension and Endocrinology, Department of Medicine, Nihon University School of Medicine, Japan and ${ }^{3}$ Kasukabe-Kisen Hospital, Japan

Received for publication May 15, 2014; Accepted for publication November 16, 2014

Correspondence to Dr. Atsushi Satomura, satomura.atsushi@nihon-u.ac.jp
} 




Figure 1. Clinical course of the eGFR (estimated glomerular filtration rate) values. The mean rate at which the glomerular filtration rate (GFR) decreased in the current patient between 45 and 65 years of age is $1.61 \mathrm{~mL} / \mathrm{min} / 1.73 \mathrm{~m}^{2} /$ year. It took approximately 20 years for the current patient to develop chronic renal failure to the point of requiring treatment with hemodialysis.

Subjects harboring a c.196G $>$ C nucleotide change (amino acid substitution: E66Q) in the GLA gene, resulting in a low GLA activity, have recently been detected at an unexpectedly high frequency (0.5-1\%) among Korean and Japanese individuals based on Fabry screening examinations (2, 7-9), which has increased interest as to whether c.196G $>$ C is a disease-causing mutation or functional polymorphism. Lee et al. (9) reported that the residual GLA activity in leukocytes obtained from patients with the E66Q mutation is 19.0$36.3 \%$ of that observed in normal controls, while the allele frequency of the E66Q mutation in the Korean population is estimated to be $1.046 \%$. Therefore, the E66Q mutation in the GLA gene may be a functional polymorphism. However, the E66Q mutation in the GLA gene was recently shown to be a genetic risk factor for cerebral small-vessel occlusion in elderly Japanese men by Nakamura et al. (10). These two studies thus obtained different outcomes regarding the presence of the E66Q mutation.

We herein report the findings of an electron microscopic examination of a renal biopsy specimen obtained from a chronic renal failure patient with the E66Q mutation in an attempt to elucidate the potential pathogenic role of the E66Q mutation in the GLA gene. We also determined whether enzyme replacement therapy could be successfully initiated in this patient.

\section{Case Report}

A 66-year-old Japanese man was suspected of having Fabry disease as a result of a screening study for Fabry disease performed in end-stage renal disease (ESRD) patients treated with chronic hemodialysis at our hospital. The plasma GLA activity in the patient was $1.2 \mathrm{nmol} / \mathrm{hour} / \mathrm{mL}$, which is lower than that observed in other patients; specifically, the GLA activity in leukocytes was $39 \%$ of the mean value noted in normal controls. The patient also had the
E66Q mutation in the GLA gene, although he did not show any clinical manifestations of Fabry disease, other than ESRD, and did not have a positive family history for the condition.

During the clinical course of the disease, the patient was admitted to a general hospital in Tokyo at 45 years of age due to edema of both lower extremities. Upon admission, he displayed massive proteinuria, with serum creatinine and blood urea nitrogen levels at the time of a renal biopsy of $1.6 \mathrm{mg} / \mathrm{dL}$ and $25 \mathrm{mg} / \mathrm{dL}$, respectively. The eGFR was 38.9 $\mathrm{mL} / \mathrm{min} / 1.73 \mathrm{~m}^{2}$, and the patient was subsequently diagnosed with interstitial nephritis based on the findings of light microscopy of an ultrasound-guided percutaneous renal biopsy specimen. Although he received treatment with prednisolone, the interstitial nephritis gradually progressed to end-stage renal failure twenty years later (Fig. 1). He therefore started hemodialysis therapy at 65 years of age (the details of his previous illness and laboratory findings are unknown). His clinical history did not indicate any clinical manifestations of Fabry disease, including angiokeratoma, acroparesthesia, hypohidrosis, cardiomegaly or corneal or lenticular opacity. The results of an electrocardiogram were within the normal range, without a high voltage. He therefore underwent transthoracic echocardiography, which showed no left ventricular hypertrophy, with an interventricular septum and left ventricular posterior wall thickness of $12 \mathrm{~mm}$ and $10 \mathrm{~mm}$, respectively. No characteristic symptoms of Fabry disease, other than end-stage renal failure, were noted.

At 45 years of age, a histological examination performed via light microscopy visualized 10 glomeruli, of which two exhibited global sclerosis. The other eight glomeruli were found to contain a mild mesangial matrix with the proliferation of mesangial cells in various portions, segmental sclerosis, including partial wrinkling of the glomerular basement membrane, and extracapillary (cellular crescent) proliferation. Scattered fibrosis surrounded the sclerotic glomeruli, and both the proximal and distal tubules were partially atrophic and infiltrated with mononuclear cells (Fig. 2). The walls of small- to medium-sized arteries displayed sclerotic degeneration with hyalinization in some parts. We subsequently assessed a renal biopsy specimen obtained from the patient using hematoxylin and eosin (Fig. 3A, B), periodic acid-Schiff (Fig. 3C, D) and periodic acid methenaminesilver (Fig. 3E, F) staining; however, no characteristic pathological findings indicating the accumulation of Gb3 (a feature of Fabry disease), such as vacuolated epithelial cells in the glomeruli, were observed.

Therefore, an electron microscopic examination was performed using the residual renal specimen obtained from the patient at 45 years of age at the general hospital. Consequently, electron microscopy revealed partial effacement of the foot processes of glomerular epithelial cells and the presence of deposits in both the mesangial and paramesangial areas of the glomeruli. However, no inclusion bodies in podocytes, endothelial or mesangial cells, tubular epithelial 


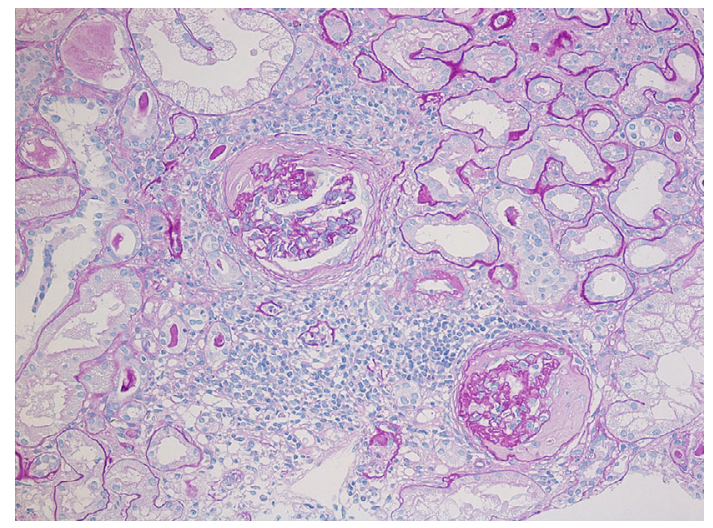

Figure 2. Light microscopic analysis of the renal biopsy specimen. Of 10 glomeruli analyzed, two displayed extracapillary (cellular crescent) proliferation. Scattered fibrosis surrounded the sclerotic glomeruli, and both the proximal and distal tubules were partially atrophic and infiltrated with mononuclear cells.

cells or interstitial capillaries (Fig. 4), indicative of the accumulation of Gb3 in patients with Fabry disease, were detected.

We suspected that the patient should not be diagnosed with Fabry disease, but rather IgA nephropathy or focal segmental sclerosis with ischemic changes, without findings for immunofluorescence histochemistry.

\section{Discussion}

Efforts have been made to explain the wide range of phenotypic heterogeneity observed in cases of Fabry disease. To date, more than 500 mutations in the GLA gene, located at $\mathrm{Xq} 22$, have been identified. The majority of these mutations are private and restricted to only one or a few families (The Human Gene Mutation Database: http://www.hgmd.cf.ac.uk/ ac/index.php). More than $58 \%$ of mutations are missense or nonsense, with the rest being splicing, small or gross deletions or insertion mutations. Most mutations noted in patients with classical Fabry disease affect the catalytic activity of the enzyme, whereas mutations in individuals with atypical Fabry disease do not affect the enzyme activity. Therefore, Fabry disease exhibits various clinical disease courses based on the type of mutation $(11,12)$.

Several previous studies have reported the prevalence of Fabry disease in patients with ESRD. For example, Kotanko et al. (13) reported an incidence of $0.16 \% \quad(n=4)$ among 2,480 Austrian dialysis patients, while Merta et al. (14) documented an incidence of $0.15 \%(n=5)$ among 3,370 Czech Republic hemodialysis patients. In Japan, various small studies have suggested a prevalence of $0.16-1.2 \%$ among ESRD patients treated with chronic hemodialysis $(2,15,16)$. Notably, Tsuboi et al. (17) reported the prevalence of Fabry disease in Japan to be 0.25 cases per 100,000 individuals. Therefore, the true prevalence of this disease may be much higher in chronic hemodialysis pa- tients than in the general population. We investigated 208 Japanese men with ESRD treated with chronic hemodialysis therapy screened for Fabry disease by measuring the plasma GLA activity. (Informed consent was obtained from all participants.) One patient demonstrated a low plasma GLA activity with a residual GLA level. Upon a further examination, the patient was shown to have the E66Q mutation in the GLA gene. Therefore, we recommend measuring the plasma GLA activity in patients with chronic kidney disease of unknown cause and/or those presenting with the clinical manifestations of Fabry disease based on their clinical history.

Several investigators have reported finding the c.196G $>C$ base change in many patients with the late-onset phenotype of Fabry disease (5-7). Of note, we found no studies reporting a c.196G >C base change in non-Asian populations when searching Pub-Med. Therefore, the E66Q amino acid substitution is considered to be pathogenic, causing late-onset Fabry disease subsequently leading to renal and cardiac disease. However, no convincing evidence for this hypothesis has been obtained to date. Recently, we reported that an ESRD patient with the E66Q mutation treated with chronic hemodialysis did not exhibit the characteristic pathological findings of Fabry disease normally detected in biopsied skin tissues (18). Furthermore, Kobayashi et al. (19) reported an ESRD patient with the E66Q mutation treated with chronic hemodialysis who did not present with the characteristic pathological findings of Fabry disease normally detected as a result of the abnormal accumulation of Gb3 in cardiac tissues. In both of these reports, electron microscopic examinations showed no pathological changes specific to Fabry disease in the biopsied tissue specimens of chronic renal failure patients with the E66Q mutation. It is thus very important that electron microscopic examinations of the renal biopsy specimen obtained from the present chronic renal failure patient with the E66Q mutation revealed no characteristic findings of Fabry disease. Furthermore, Lee et al. (9) studied 1,176 alleles in the Korean population and detected the GLA gene E66Q mutation in 12 alleles. The authors estimated the frequency of alleles with the E66Q mutation in the Korean population to be $1.046 \%$. The expression of the GLA gene with the E66Q mutation is characterized by the transient overexpression of related proteins in COS-7 cells, accounting for $43.8 \%$ of the normal mean activity, and is efficiently expressed in lysosomes. Lee et al. further demonstrated that white blood cells derived from subjects with the E66Q enzyme display an activity $19.0-30.3 \%$ that of normal and concluded that the E66Q mutation is a functional polymorphism. However, the GLA gene E66Q mutation is a genetic risk factor for cerebral small-vessel occlusion in elderly Japanese men, according to Nakamura et al. (10). We previously stated that it is questionable whether detecting the E66Q mutation in the GLA gene is adequate for predicting cerebral small-vessel occlusion (20). A study by Nakamura et al. (10) used newborns as a control group. However, newborns generally do not exhibit cerebral small-vessel oc- 

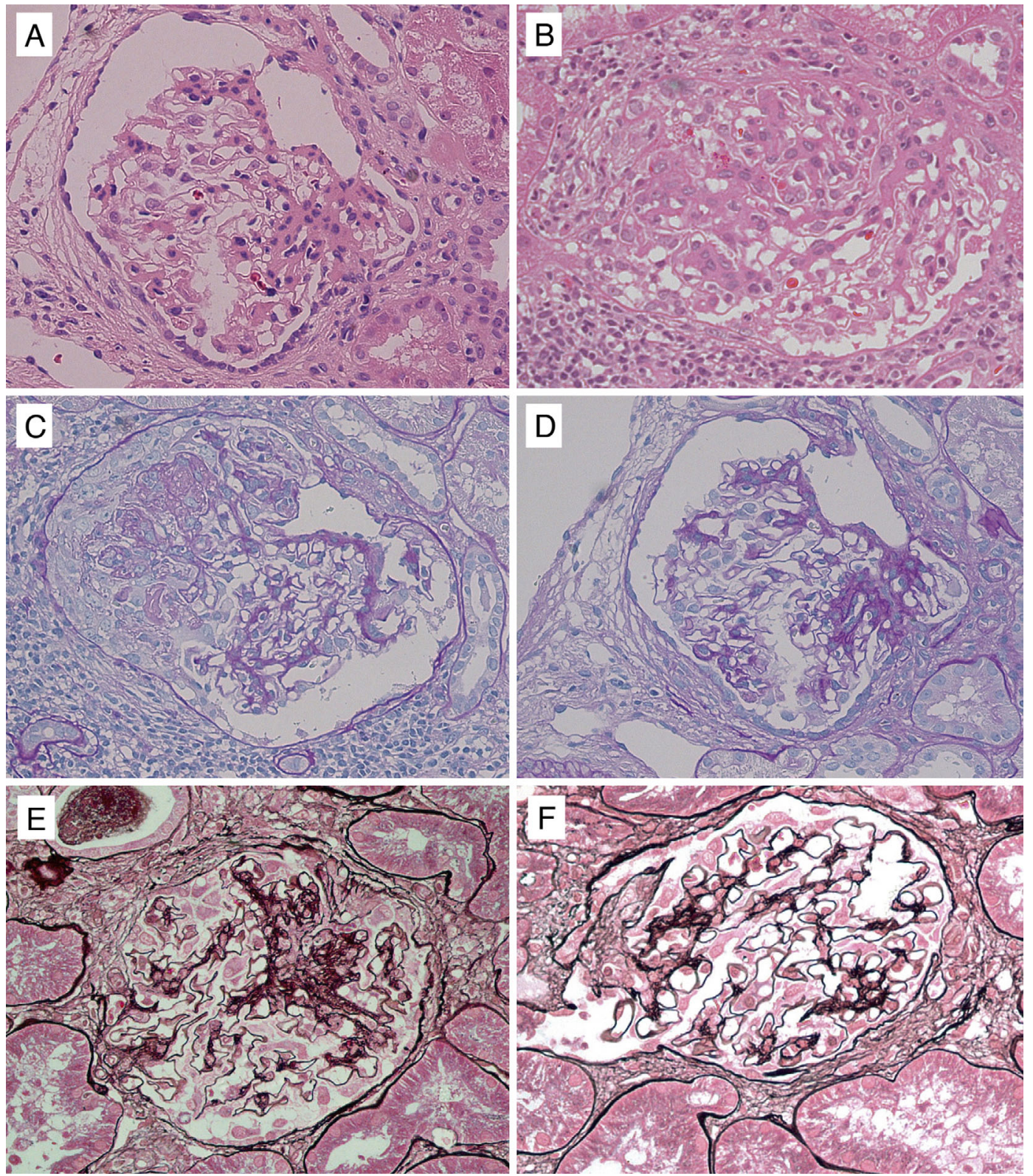

Figure 3. Light microscopic analysis of the renal biopsy specimen. The glomeruli developed mild mesangioproliferative glomerulonephritis with cellular crescent formation, ischemic changes and mild tubular injury. No characteristic pathological findings of vacuolated epithelial cells in the glomeruli suggestive of Fabry disease were noted, as observed on Hematoxylin and Eosin staining (A, B), periodic acid-Schiff (C, D) and periodic acid methenamine-silver (E, F) staining.

clusion, although they may develop this complication in the future. Therefore, the use of such a control group makes it difficult to obtain convincing data for a statistical analysis.

Tøndel et al. (21) reported that glomerular and vascular changes are noted in biopsied renal specimens prior to progression to overt proteinuria and a decreased GFR. The current patient had significant proteinuria in his urine and demonstrated a decreased renal function. Unfortunately, we were unable to perform an immunohistochemistry examination using monoclonal anti-Gb3 antibodies to assess the accumulation of Gb3 in the kidneys due to the small quantity of residual renal biopsy tissue. If the patient had indeed had Fabry disease, the renal biopsy would have shown changes associated with Gb3 accumulation. Furthermore, the patient's leukocyte GLA activity level was decreased by $39 \%$ compared with that observed in normal controls, but higher than that seen in patients with classic Fabry disease. Hence, the reduction in activity caused by the E66Q mutation may not have been sufficient to induce $\mathrm{Gb} 3$ accumulation in the tissues. Moreover, the finding of a lyso-Gb3 level below the limit of detection of $2 \mathrm{nmol} / \mathrm{L}$, as noted in normal controls, may not be adequate to cause Fabry disease. Moreover, it took approximately 20 years for the current patient to develop chronic renal failure to the point of requiring hemodialysis (Fig. 1). Branton et al. (5) reported that the mean change in GFR after the onset of chronic renal insufficiency 


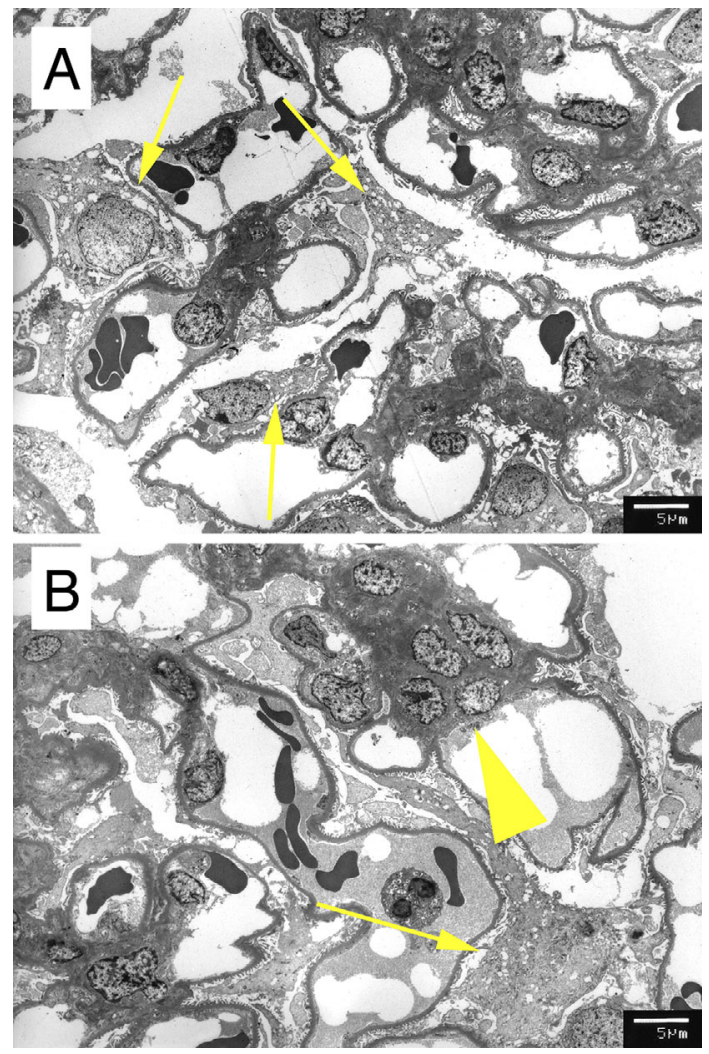

Figure 4. Electron microscopic analysis of the renal biopsy specimen. The electron micrograph analysis did not show any inclusion bodies in podocytes (yellow arrows in both $\mathbf{A}$ and $B$ ), endothelial or mesangial cells, tubular epithelial cells or interstitial capillaries. Electron micrography revealed the presence of a few paramesangial and mesangial deposits in the glomeruli (yellow arrowhead in B).

(Each bar indicates $5 \mu \mathrm{m}$, respectively).

is $-12.2 \mathrm{~mL} / \mathrm{min}$ per year, with patients usually reaching end-stage renal disease after 4.1 years. The present patient did not experience the typical clinical course of Fabry disease.

In order to confirm whether the E66Q mutation is a functional polymorphism, it is necessary to perform pathological studies, particularly focusing on the detection of Gb3 accumulation. In the present study, we performed enzymatic, genetic and pathological studies in a patient with the E66Q mutation in the GLA gene. We also performed additional pathological analyses to assess the role of the E66Q mutation in the pathogenesis of chronic kidney disease. The findings of the renal biopsy specimens, together with the presence of chronic kidney disease, may support the hypothesis that the E66Q mutation in this patient was a functional polymorphism.

The E66Q mutation in the GLA gene is thought to be a functional polymorphism, at least in the present case. When considering a diagnosis of Fabry disease in patients with the E66Q mutation, it is therefore necessary to avoid the unnecessary initiation of enzyme replacement therapy.

The authors state that they have no Conflict of Interest (COI).

\section{References}

1. Germain DP. Fabry disease. Orphanet J Rare Dis 5: 30, 2010.

2. Nakao S, Kodama C, Takenaka T, et al. Fabry disease: detection of undiagnosed hemodialysis patients and identification of a "renal variant" phenotype. Kidney Int 64: 801-807, 2003.

3. Nakao S, Takenaka T, Maeda $M$, et al. An atypical variant of Fabry's disease in men with left ventricular hypertrophy. N Engl J Med 333: 288-293, 1995.

4. Mehta A, Clarke JT, Giugliani R, et al. Natural course of Fabry disease: changing pattern of causes of death in FOS-Fabry Outcome Survey. J Med Genet 46: 548-552, 2009.

5. Branton MH, Schiffmann R, Sabnis SG, et al. Natural history of Fabry renal disease. Influence of $\alpha$-galactosidase A activity and genetic mutations on clinical course. Medicine 81: 122-138, 2001.

6. Feriozzi S, Torras J, Cybulla M, et al. The effectiveness of longterm agalsidase alfa therapy in the treatment of Fabry nephropathy. Clin J Am Soc Nephrol 7: 60-69, 2012.

7. Shimotori M, Maruyama H, Nakamura G, et al. Novel mutations of the GLA gene in Japanese patients with Fabry disease and their functional characterization by active site specific chaperone. Hum Mutat 29: 331, 2008.

8. Yoshitama T, Nakao S, Takenaka T, et al. Molecular genetic, biochemical, and clinical studies in three families with cardiac Fabry's disease. Am J Cardiol 87: 71-75, 2001.

9. Lee BH, Heo SH, Kim GH, et al. Mutations of the GLA gene in Korean patients with Fabry disease and frequency of the E66Q allele as a functional variant in Korean newborns. J Hum Genet 55: 512-517, 2010.

10. Nakamura K, Sekijima Y, Nakamura K, et al. p.E66Q mutation in the GLA gene is associated with a high risk of cerebral smallvessel occlusion in elderly Japanese males. Eur J Neurol 21: 4956, 2014.

11. Ashton-Prolla P, Tong B, Shabbeer J, Astrin KH, Eng CM, Desnick JR. Fabry disease: twenty-two novel mutations in the alpha-galactosidase A gene and genotype/phenotype correlations in severely and mildly affected hemizygotes and heterozygotes. J Investing Med 48: 227-235, 2000.

12. Germain DP. A new phenotype of Fabry disease with intermediate severity between the classical form and the cardiac variant. Contrib Nephrol 136: 234-240, 2001.

13. Kotanko P, Kramar R, Devrnja D, et al. Results of a nationwide screening for Anderson-Fabry disease among dialysis patients. J Am Soc Nephrol 15: 1323-1329, 2004.

14. Merta M, Reiterova J, Ledvinova J, et al. A nationwide blood spot screening study for Fabry disease in the Czech Republic haemodialysis patient population. Nephrol Dial Transplant 22: 179-186, 2007.

15. Tanaka M, Ohashi T, Kobayashi M, et al. Identification of Fabry's disease by the screening of alpha-galactosidase A activity in male and female hemodialysis patients. Clin Nephrol 64: 281-287, 2005.

16. Ichinose M, Nakayama M, Ohashi T, et al. Significance of screening for Fabry disease among male dialysis patients. Clin Exp Nephrol 9: 228-232, 2005.

17. Tsuboi K, Suzuki S, Nagai M. Descriptive epidemiology of Fabry disease among beneficiaries of the specified disease treatment research program in Japan. J Epidemiol 22: 370-374, 2012.

18. Togawa $T$, Tsukimura $T$, Kodama $T$, et al. Fabry disease: Biochemical, pathological and structural studies of the $\alpha$ galactosidase A with E66Q amino acid substitution. Mol Genet Metab 105: 615-620, 2012.

19. Kobayashi M, Ohashi T, Fukuda T, et al. No accumulation of globotriaosylceramide in the heart of a patient with the E66Q mutation in the $\alpha$-galactosidase A gene. Mol Genet Metab 107: 711$715,2012$. 
Intern Med 54: 1819-1824, 2015 DOI: 10.2169/internalmedicine.54.3279

20. Satomura A, Yanai M, Fujita T, Nakayama T. Comment on: p.E66 $\mathrm{Q}$ mutation in the GLA gene is associated with a high risk of cerebral small-vessel occlusion in elderly Japanese males. Eur $\mathbf{J}$ Neurol 21: e62, 2014.
21. Tøndel C, Bostad L, Hirth A, Svarstad E. Renal biopsy findings in children and adolescents with Fabry Disease and minimal albuminuria. Am J Kidney Dis 51: 767-776, 2008.

(C) 2015 The Japanese Society of Internal Medicine http://www.naika.or.jp/imonline/index.html 